This is a post-peer-review, pre-copyedit version of an article published in Neural Computing Applications.

The final authenticated version is available online at: http://dx.doi.org/10.1007/s00521-013-1364-4

Neural Computing Applications manuscript No.

(will be inserted by the editor)

\title{
An Experimental Characterization of Reservoir Computing in Ambient Assisted Living Applications
}

\author{
Davide Bacciu • Paolo Barsocchi • \\ Stefano Chessa • Claudio Gallicchio • \\ Alessio Micheli
}

Received: date / Accepted: date

\begin{abstract}
In this paper, we present an experimental introduction and critical evaluation of a Reservoir Computing (RC) approach for Ambient Assisted Living (AAL) applications. Such an empirical analysis jointly addresses the issues of efficiency, by analyzing different system configurations towards the embedding into computationally constrained wireless sensor devices, and of efficacy, by analyzing the predictive performance on real-world applications. First, the approach is assessed on a validation scheme where training, validation and test data are sampled in homogeneous ambient conditions. Then, it is introduced an external test set involving a new setting, i.e. a novel ambient, which was not available in the first phase of model training and validation. The specific testbed considered in the paper allows us to investigate the capability of the RC approach to discriminate among user movement trajectories from RSSI sensor signals. This capability can be exploited in various AAL applications targeted at learning user indoor habits, such as in the proposed indoor movement forecasting task. Such a joint analysis of the efficiency/efficacy tradeoff provides novel insight in the concrete successful exploitation of Reservoir Computing for AAL tasks and for their distributed implementation into wireless sensor networks.
\end{abstract}

Keywords Ambient Assisted Living · Reservoir Computing · Wireless Sensor Networks · Indoor User Movement Forecasting

D. Bacciu, S. Chessa, C. Gallicchio, A. Micheli

Dipartimento di Informatica - Universita di Pisa

Tel.: +39-050-2212717

Fax: +39-050-2212726

E-mail: \{bacciu,ste,gallicch,micheli\}@di.unipi.it

P. Barsocchi

Istituto di Scienze e Tecnologie dell'Informazione, Consiglio Nazionale delle Ricerche

E-mail: paolo.barsocchi@isti.cnr.it

(c) 2019. This manuscript version is made available under the CC-BY-NC-ND 4.0 license http://creativecommons.org/licenses/ by-nc-nd/4.0/ 


\section{Introduction}

Ambient Assisted Living (AAL) (AAL, 2009) is a recent research program issued by the European Community. It addresses those technologies that can be used to improve the quality of life of elders or disabled by assisting them and making them feel secure and protected in the places where they live or work. The AAL effort is strongly motivated by the fact that the number of elders in the European Community is constantly increasing, and a large demand of AAL-related technologies is expected in the near future. AAL applications typically rely on a number of devices, equipped with sensing capabilities that provide data which serve to detect events occurring around or to the user. In order to facilitate the user acceptance of AAL technologies, these devices, typically referred to as sensors, should be of reduced size (especially those deployed on the user itself) and make large use of wireless communication technologies. A Wireless Sensor Network (WSN) for AAL comprises a variable number of tiny sensors, each of which is a micro system embedding a processor/memory subsystem, a set of transducers, a radio subsystem and a battery. Sensors can self-organize in order to build and maintain a wireless network for their communications.

A WSN can deliver consistent amounts of data to an AAL system, providing a very crude picture of the environment/user being monitored. As such, consistent refinement of the sensed data is needed in order to extract the highlevel information, e.g. user activities or events, that is best suited to processing within an AAL system. Given the peculiarities of the information involved, this process requires an approach characterized by adaptivity and robustness to input noise. Within this scenario, Machine Learning models, in general, and Neural Networks, in particular, provide feasible data-driven solutions. We focus on Recurrent Neural Networks (RNNs) (Kolen and Kremer, 2001), representing dynamical neural network models capable of directly processing the temporal streams of sensed data produced by the nodes of the WSN. Within such RNN class, we consider the Reservoir Computing (RC) paradigm (Verstraeten et al, 2007; Lukosevicius and Jaeger, 2009) and, more specifically, the Echo State Network (ESN) model (Jaeger and Haas, 2004; Jaeger, 2001). ESN feature an interesting trade-off between extreme computational efficiency and the ability to learn in temporal sequence domains.

In view of a complete integration between the devices of a WSN and the learning capabilities offered by the RC networks, we envisage a system in which the learning models are embedded directly on-board the nodes of the WSN. However, learning in distributed WSN systems for AAL tasks raises novel challenges related to the effectiveness and efficiency in treating the sensed (temporal) data. RC has the potential for providing learning solutions that are effective in terms of time-series prediction accuracy, given the RC ability of capturing complex time-dependent behaviors in its reservoir state, yet efficient from the point of view of computational and memory requirements. As such, they are good candidates for the development of computational learning models that can be embedded on the WSN devices. Such devices have 
limited computational capabilities, but provide direct access to sensor data, either acquired by the on-board transducers or received from neighboring sensors through the wireless radio. An embedded RC model can thus be trained to provide an on-board preprocessing of the sensorial information to predict refined information concerning the environment/user state. This, on the one hand, reduces the communication burden, since only high-level predictions are transferred to the (typically centralized) AAL system through the wireless communication media. On the other hand, it allows to distribute the computational burden, relieving the central AAL system from the processing of the low-level sensor information.

The deployment of such an innovative distributed embedded learning system, needs to be anticipated by an empirical assessment of the RC neural models, to determine their suitability in addressing AAL-related tasks. Current works in literature propose experimental analyses that are fairly limited in scope and, basically, found on the sole use of artificial data. In this concern, a benchmarking of the RC approach is still needed, with particular regard to the assessment of the effectiveness on real-world data and the evaluation of the efficiency of the learning models. To this aim, we consider a computational learning task that follows from a representative AAL problem, i.e. the prediction of the indoor user spatial context in the monitored environment. This is one of the most critical information required by an AAL system, which is essential to deliver the services to the user in the appropriate place, and at the right time. The user trajectory information can be easily collected, in the form of time-series, by measuring the Radio Signal Strength (RSS) among a few wireless devices deployed in the environment and a user wearable sensor. As it will be discussed in Section 2, such real-world task involves streams of noisy input data and requires the ability to appropriately adapt to specific user/environment conditions.

Motivated by the above-mentioned RSS-localization challenges and, more in general, by the study of the design of WSN-embedded learning systems for AAL tasks, we propose a novel RC approach to indoor movement forecasting, that aims at predicting the future user context based on real-world noisy RSS input data. This work presents a systematic experimental investigation of the $\mathrm{RC}$ approach that jointly considers both the efficiency and the efficacy of proposed solution in a real-life AAL application. A systematic assessment of the $\mathrm{RC}$ approach requires solid data, gathered on a realistic scenario that is consistent with the task to be addressed. To this end, we have devised a real-life localization benchmark, comprising a small WSN network capturing localization information in different experimental premises, and whose details are given in Section 4.1. The proposed benchmark focuses on learning to discriminate among different types of user trajectories based on RSS measurements across time. Such task is associated to a specific semantics in AAL applications, related to the forecasting of the user spatial context, i.e. room change vs. room preservation. This is intended to be a concrete use case for an RC approach capable of addressing diverse AAL tasks associated to learning user indoor activity habits. A through evaluation cannot abstract from an appro- 
priate validation scheme, tailored to the identification of the major properties of the learning model with respect to the application at hand. In this sense, it is worth to evaluate the ability of the RC approach in generalizing its prediction performance to novel, unknown, environments. Such property is needed to show that, while the proposed solution can increase the level of service personalization (i.e. by making accurate prediction of the user spatial context), it can, at the same time, yield to a reduction of the setup and installation costs by means of adequate generalization capabilities. In view of a distributed embedded realization of the neural system, efficiency is a relevant desirable property that should be considered and assessed. Hence, in this paper, we specifically evaluate the trade-off between predictive accuracy and memory occupation cost, which is central to embed learning modules on low-power WSN devices. In this sense, the RC paradigm offers an interesting ground due to its intrinsic characteristic of efficiency: in this paper, we discuss an empirical assessment of the relation between the performance and the implementation cost of constrained RC networks. In particular, using a lightweight scheme for storing the untrained reservoir weights, we consider the effect of reservoir dimensionality, which has a direct impact on both predictive accuracy and memory occupation cost. Additionally, we explore the performance of modular neural architectures, coherent with the WSN distributed structure, comprising independent learning modules that can share information through the WSN communication media. Various degrees of learning interactions are discussed and experimentally assessed, including cases of full and partial connectivity, as well as committee architectures ${ }^{1}$.

The remainder of the paper is articulated as follows: Section 2 discusses the background on RSS-based localization and available learning-based solutions; Section 3 describes the detail of the RC model, while Section 4 discusses the localization benchmark and the results of the experimental assessment; Section 5 concludes the paper.

\section{Background}

AAL applications normally operate in indoor environments and require localization services. However, these services can hardly be supported by established global positioning systems (such as GPS or Galileo) that do not provide the appropriate accuracy in indoor environments. For this reason, localization information is generally obtained by means of other technologies, based on

\footnotetext{
1 This paper, based on ex-novo conducted experiments, extends the preliminary investigations presented in the conference papers (Gallicchio et al (2012); Bacciu et al (2011)), proposing a comprehensive vision of the costs that can practically occur for real deployment of the RC models into mote devices. In particular, previous works did not include an exhaustive comparative analysis of different network sizes and configurations (Gallicchio et al (2012); Bacciu et al (2011)), or the adoption of a reduced reservoir weight encoding scheme (Gallicchio et al (2012); Bacciu et al (2011)) or the external performance evaluation (Gallicchio et al (2012)).
} 
sensors or cameras deployed in the user environment. In the attempt to deliver cost-effective localization services, researchers tend to privilege the use of widely available technical solutions exploiting off-the-shelves components, and generally avoid the use of cameras, that may present user acceptance issues (EvAAL, 2011), as they are more invasive on the user life.

A practical solution for indoor user localization consists in using a number of fixed, environmental sensors whose position is known (these sensors are called anchors) and a wearable sensor (also called mobile sensor) that is constantly carried by the user. The anchors and the mobile frequently exchange radio packets (called beacons) and, for each beacon, they measure the corresponding strength of the radio signal (called Radio Signal Strength, RSS). Since the strength of the signal decays with the distance between the source and the receiver, it is possible to relate a RSS associated to a beacon, with the distance between the anchor and the mobile that exchanged that beacon. Hence, a localization system can, in principle, exploit the RSS information collected over a period of time, to predict an approximated user position. Unfortunately, the propagation of the radio signals in indoor environments is subject to phenomena that may alter significantly the RSS measure depending on the specific setting of each room. Additionally, the RSS measurements are greatly affected by the relative position of the user with respect to the line of sight between the anchor and the mobile. Nevertheless, RSS-based localization is a cost-effective solution for localization of users in indoor AAL applications, due to the widespread deployment of wireless networks and WSN infrastructures that make available RSS measurements. Mainly, we distinguish between two alternative computational approaches to localize users leveraging the RSS measurements, i.e. model-based and fingerprinting positioning.

Model-based positioning is popular approach in literature that founds on expressing radio frequency signal attenuation using specific path loss models (Barsocchi et al, 2009b,a). Given an observed RSS measurement, these methods triangulate the person based on distance calculations from multiple anchors. However, the relationship between the user position and the RSS information is highly complex and can hardly be modeled due to multipath, metal reflection, and interference noise. Thus, RSS propagation may not be adequately captured by a fixed invariant model. In these conditions, it is not surprising that such analytical models fail to reach acceptable accuracy levels, with typical localization errors ranging between 2 to 4 meters (depending on the setting) (EvAAL, 2011).

Differently from model-based approaches, fingerprinting techniques, such as (Bahl and Padmanabhan, 2000; Youssef and Agrawala, 2005; Kushki et al, 2007; Pan et al, 2006), create a radio map of the environment based on RSS measurements at known positions by means of an offline map-generation phase. Clearly, the localization performance of fingerprinting-based model relies heavily on the choice of the distance function that is used to compute the similarity between the RSS measured in the online phase, with the known RSS fingerprints. Further, the offline generated ground truth might need to be revised in 
case of changes to the room/environment configuration, which might result in relevant discrepancies in the known fingerprints.

Adaptivity and flexibility with respect to minor environmental changes can be introduced in the fingerprinting approach, by exploiting computational learning models to infer the relationship between the RSS samples and the subject location. The majority of the approaches in literature exploit probabilistic learning techniques to learn a posterior estimate of user location given RSS measurements at known location, e.g. (Zàruba et al, 2007) and (Liu et al, 2007). However, probabilistic models have consistent computational costs connected both with the learning and the inference phase, which might grow exponentially with the number of sensors in the area. k-Nearest Neighbor (kNN) algorithms often show good accuracy which, however, tends to be particularly sensitive to beacon positioning (Luo et al, 2011). Nevertheless, kNN is characterized by well-known expensive memory and computational requirements which make it less suitable for embedding in WSN nodes. Similar complexity considerations apply to the use of Multi Layer Perceptrons (Battiti et al, 2002) and Support Vector Machines (Brunato and Battiti, 2005; Wu et al, 2004) in location fingerprinting, that is mainly limited to wireless local area networks of powerful access points and palmtop devices.

The measurement of RSS values over time provides information on the subject trajectory under the form of a time series of sampled signal strength, which allows for subject tracking rather than simple localization. However, neither the static RSS-map of the fingerprinting approaches discussed so far, nor the predefined propagation model of model-based solutions are appropriate to capture such dynamic knowledge. The adaptive fingerprinting approaches discussed above, for instance, do little to exploit the sequential nature of the RSS streams, whereas they provide static pictures of the actual state of the environment. More importantly, these localization approaches can only provide the estimate of the current user position, but lack the ability of forecast his/her future location. Being capable of predicting the future user context is of a great added value in AAL applications, since it enhances service reactivity and personalization. In this respect, there exist several machine learning models capable of explicitly dealing with signals characterized by such time-dependent dynamics including, for instance, probabilistic Hidden Markov Models (HMM), RNN (Kolen and Kremer, 2001) and kernel methods for sequences (Gärtner, 2003). Kontkanen et al (2004), for instance, discuss the application of HMMs to tracking using RSS information: their use in computationally-constrained devices is, however, limited by the complexity of the inference process needed at test time. Similar problems occur in Kernel Methods for sequences, whose cost scales at least quadratically with the input length, e.g. Gärtner (2003).

Echo State Networks (ESNs) (Jaeger and Haas, 2004; Jaeger, 2001) are an RC model well suited for sequence processing. Their most striking feature is efficiency: training is limited to the linear outputs whereas the dynamic part of the network is fixed. Additionally, the cost of input encoding scales linearly with the length of the sequence for both training and test, comparing favorably with competitive state-of-the-art learning models for sequence do- 
mains. Recently, ESNs have shown good potential in a range of tasks related to autonomous systems modeling, also by virtue of their marked efficiency. Examples include event detection and localization in autonomous robot navigation (Antonelo et al, 2008, 2007), multiple robot behavior modeling and switching (Waegeman et al, 2009), robot behavior acquisition (Hartland and Bredeche, 2007) and robot control (Oubbati et al, 2010). However, such applications are mostly focused on modeling robot behaviors and often use artificial data obtained by simulators (e.g. (Antonelo et al, 2008, 2007; Waegeman et al, 2009)). More recently, Gallicchio et al (2012) have proposed an ESN application to user's indoor movements forecasting using real-world data, followed by a preliminary investigation of its generalization ability to unseen ambient configurations by Bacciu et al (2011), where, however, efficiency issues are not taken into consideration. Further differences with respect to these previous approaches are summarized in Section 1 (see in particular footnote 1).

The latter works demonstrates the potential of the ESN approach for RSSbased tracking, but miss a systematic performance evaluation, especially with regard to the constraints and possible configurations imposed by a distributed low-power environment such as WSN. In the following, we provide a thorough empirical assessment of several distributed ESN configurations, based on realistic WSN-induced layouts, with application to user movement forecasting using real-world RSS data, in both homogeneous and heterogeneous validation settings (see Section 4).

\section{Reservoir Computing and Echo state Networks}

Reservoir Computing (RC) (Verstraeten et al, 2007; Lukosevicius and Jaeger, 2009) represents an increasingly popular paradigm for efficiently modeling Recurrent Neural Networks (RNNs). RC networks implement dynamical systems, and are essentially based on the separation between a recurrent dynamical reservoir and a non-recurrent readout. The role of the reservoir is to encode the history of the input signals which drive the system. At each time of computation, such network state provides the readout with a rich set of "reservoir" dynamics, from which to linearly combine in order to compute the output. The outstanding feature of $\mathrm{RC}$ is the extreme efficiency of training. Indeed, the simple readout component is the only trained part of the network, while the reservoir is left untrained after contractive initialization. RC comprises several approaches, including the popular Echo State Networks (ESNs) (see Jaeger and Haas (2004); Jaeger (2001)), Liquid State Machines (see Maass et al (2002)) and BackPropagation Decorrelation (Steil, 2004, 2006), among the others. In particular, in this paper we focus on the ESN model.

An ESN is composed of an input layer with $N_{U}$ units, a reservoir layer with $N_{R}$ units and a readout with $N_{Y}$ units (see Figure 1). The reservoir is a large, sparsely-connected, non-linear, recurrent layer, which is used to perform a contractive encoding of the history of driving input signals into a state space. Typically, the non-linearity adopted as activation function of reservoir units 


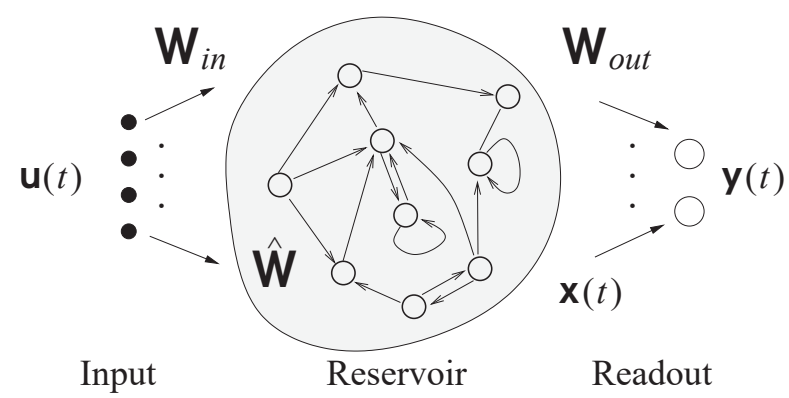

Fig. 1 Architecture of an ESN with $N_{U}=4$ input units, $N_{R}=9$ reservoir units and $N_{Y}=2$ readout units.

is the hyperbolic tangent tanh. The readout is a layer of linear units, used to compute the output of the ESN by a linear combination of the reservoir units' activations. In particular, in this work, we take into consideration a variant of the standard ESN model, called the leaky integrator ESN (LI-ESN) (see Jaeger et al (2007); Lukosevicius and Jaeger (2009)). In LI-ESNs, standard sigmoidal reservoir units are replaced by leaky integrator units, which apply an exponential moving average to reservoir state values and imply a better handling of input sequences that change slowly with respect to the frequency of sampling (Lukosevicius and Jaeger, 2009; Antonelo et al, 2008). In practice, LI-ESNs result in an RC model particularly suitable for the characteristics of RSS input signals, as shown in Gallicchio et al (2012) through real-world experiments.

In the following, $\mathbb{R}^{N_{U}}, \mathbb{R}^{N_{R}}$ and $\mathbb{R}^{N_{Y}}$ denote respectively the input, reservoir and output spaces. The input at pass $t$ is denoted by $\mathbf{u}(t) \in \mathbb{R}^{N_{U}}$, while an input sequence of length $n$ is denoted as $s=[\mathbf{u}(1), \ldots, \mathbf{u}(n)]$. Moreover, reservoir state and output of the ESN are denoted respectively by $\mathbf{x}(t) \in \mathbb{R}^{N_{R}}$ and $\mathbf{y}(t) \in \mathbb{R}^{N_{Y}}$. At each time step $t$, the reservoir of LI-ESN computes the following state transition function:

$$
\mathbf{x}(t)=(1-a) \mathbf{x}(t-1)+a f\left(\mathbf{W}_{i n} \mathbf{u}(t)+\hat{\mathbf{W}} \mathbf{x}(t-1)\right)
$$

where $\mathbf{W}_{\text {in }} \in \mathbb{R}^{N_{R} \times N_{U}}$ is the input-to-reservoir weight matrix (possibly including also a bias term), $\hat{\mathbf{W}} \in \mathbb{R}^{N_{R} \times N_{R}}$ is the recurrent reservoir weight matrix, $f$ is a component-wise applied activation function for the reservoir units (we use $f \equiv \tanh$ ) and $a$ is a leaking rate which controls the speed of LI-ESN state dynamics $^{2}$. The leaking rate $a$ takes values in the range $[0,1]$, where larger values of $a$ imply faster state dynamics. Observe that for $a=1$, the LI-ESN state transition function in equation 1 reduces to the case of standard ESN.

2 Here we refer to the general usage of the leaking parameter $a$ in the RC literature. Note however, that equation 1 represents a reduced form of the complete leaky integrator dynamics (see e.g. Jaeger et al (2007); Jaeger (2001); Lukosevicius and Jaeger (2009)). In this regard, the characterization of equation 1 is that of a low-pass filter with smoothing parameter $a$. 
Reservoir parameters are left untrained after a random initialization subject to the constraints given by the so called echo state property (ESP) (Jaeger, 2001). The ESP essentially requires that the state of the network asymptotically depends on the driving input signal and any dependency on initial conditions is progressively lost. A sufficient condition and a necessary condition for the ESP to hold are given in literature (see e.g. Jaeger (2001)). The reservoir initialization conditions are related to a contractive setting of the reservoir state transition function, which has the effect of bounding the ESN state dynamics within a region of the state space characterized by interesting Markovian properties (see Tiňo et al (2004); Gallicchio and Micheli (2011, 2010)). For practical applications, the sufficient condition is often considered too restrictive and the necessary condition is typically used. For the case of LI-ESN, the necessary condition for the ESP can be formulated as follows:

$$
\rho(\tilde{\mathbf{W}})<1
$$

where $\tilde{\mathbf{W}}=(1-a) \mathbf{I}+a \hat{\mathbf{W}}$, and $\rho(\tilde{\mathbf{W}})$ is the spectral radius of $\tilde{\mathbf{W}}$, i.e. the largest among the absolute values of the eigenvalues of $\tilde{\mathbf{W}}$. The reservoir initialization procedure then consists in a random choice of the weight values in $\mathbf{W}_{\text {in }}$ from a uniform distribution over $\left[-\right.$ scale $_{i n}$, scale $\left._{i n}\right]$ (where scale $_{i n}$ is an input scaling parameter), and a random choice of the weight values in $\hat{\mathbf{W}}$ (typically from a uniform distribution in $[-1,1]$ ), after which $\hat{\mathbf{W}}$ is scaled so that equation 2 holds. In practice, values of $\rho$ close to 1 are used, leading to state dynamics close to the edge of chaos (Legenstein and Maass, 2007), and often resulting in good performances in applications (e.g. Jaeger (2001)).

The output of the LI-ESN is computed by the readout through a linear combination of of the reservoir units activations. Of particular interest for this paper, it is the case of binary classification tasks on sequences. In this case, the network produces one output in correspondence to each sequence, hence the readout is applied only when the input sequence has been completely seen and the encoding process by the reservoir has terminated. Accordingly, given an input sequence $\mathbf{s}$ of length $n$, the readout computes

$$
y(\mathbf{s})=\operatorname{sgn}\left(\mathbf{W}_{\text {out }} \mathbf{x}(n)\right)
$$

where $\mathbf{W}_{\text {out }} \in \mathbb{R}^{N_{Y} \times N_{R}}$ is the reservoir-to-readout weight matrix (possibly including a bias term) and $s g n$ is the sign threshold function, such that $y(s) \in$ $\{-1,+1\}$. The weight values in $\mathbf{W}_{\text {out }}$ are typically trained using efficient linear methods such as Moore-Penrose pseudo-inversion and ridge regression (see e.g. Lukosevicius and Jaeger (2009)).

The reservoir dimensionality is an RC hyper-parameter with a strong influence on both the network performance and the computational cost. While larger reservoirs often lead to better predictive performances in applications (e.g. Verstraeten et al (2007); Gallicchio and Micheli (2011)), the cost of the ESN implementation grows with the reservoir dimension both in time and in memory. In particular, due to the sparsity of reservoir units connectivity, the cost can be linear assuming a fixed maximum number of connections for each 
reservoir unit, or it is quadratically bounded more in general. This point assumes an even greater relevance in sight of the embedding of the RC networks directly on-board the devices of a WSN. Such a lightweight implementation of the reservoir network modules on the sensors represents a challenging design target (e.g. see Chang et al (2009)), due to the severe computational constraints involved (WSN sensors are equipped with a very small amount of total RAM memory, typically in the range of 8-10 Kbytes), and requires a careful assessment of the trade-off between the implementation cost and the predictive performance of the $\mathrm{RC}$ networks in real-world scenarios. The relation between the effectiveness and the memory occupation cost of RC networks for AAL tasks is investigated in this paper by experimentally evaluating the effect of reservoir dimensionality on the accuracy achieved by the LI-ESN model on a real-world task described in Section 4. Moreover, we adopt an efficient strategy for the memorization of the weight values in matrices $\mathbf{W}_{\text {in }}$ and $\hat{\mathbf{W}}$, using a small finite weight alphabet for the non-zero weight values, which can thereby be encoded using only a few bits. For instance, only 3 bits per weight are required if we consider a weight alphabet with 8 possible values.

Note that the use of a small finite alphabet of weight values for the connections pointing to the reservoir, can be traced back to the early works in RC literature (see e.g. Jaeger (2001)). In this paper, this reservoir initialization procedure is exploited with the challenging objective of an extremely efficient implementation of the RC modules directly on-board the sensors of a WSN. Although a study of the architectural pattern of connectivity among the reservoir units is out of the scope of our paper, the proposed efficient weight encoding scheme can be related and applied also to architectural variants of the standard ESN model, such as reservoir architectures based on permutation matrices (see e.g. Boedecker et al (2009); Hajnal and Lorincz (2006)), self-recurrent connections only (see e.g. Gallicchio and Micheli (2011); Fette and Eggert (2005)), or cyclic patterns of connectivity (see e.g. Rodan and Tiňo (2011)). Finally, it is interesting to observe that the proposed light-weighted encoding scheme for the reservoir weight connections, in a sense results in a quantization of the space of weight values for the reservoir part of the RC model. Although not directly connected to this aspect, related literature approaches also consider the quantization of reservoir state spaces (see e.g. Tiňo and Dorffner (2001); Büsing et al (2009)).

\section{Benchmarking RC in Indoor User Movement Prediction}

In the following, we describe a benchmark for adaptive user movement prediction using RSS traces in a real-life office scenario. First, in Section 4.1, it is provided a brief discussion of the wireless technology involved, together with a detailed description of the experimental indoor environment. In Section 4.2, the RC approach described in Section 3 is empirically assessed on the proposed forecasting benchmark, simulating different levels of information sharing among the nodes in the WSN. 
Table 1 Geometry of the area involved in the three experiments.

\begin{tabular}{c|c|c}
\hline Dataset Number & length [m] & width [m] \\
\hline 1 & 4.5 & 12.6 \\
2 & 4.5 & 13.2 \\
3 & 4 & 12.6 \\
\hline
\end{tabular}

\subsection{A Real-life Movement Prediction Benchmark}

In order to establish an effective movement prediction benchmark based on real-world data, we performed an extensive measurement campaign in the offices of the ISTI institute of CNR in the Pisa Research Area, in Italy. The area used for the measurements consists of 6 rooms with different geometry.

Each data acquisition campaign, hereafter referred to as experiment, involves two rooms (referred to as Room 1 and Room 2 in the following) with fronting doors divided by an hallway. Figures 2.a to 2.c show the layout of the 3 room couples that have been used in the campaigns. The benchmark comprises data from three separate experiments, each corresponding to one of the room couples in Figure 2, and that provide three different datasets. The area of interest for each experiment ranges from $50 \mathrm{~m}^{2}$ to $60 \mathrm{~m}^{2}$ : Table 1 provides the geometry of the indoor surfaces covered in each experiment.

It is worth to note that the measurement campaign was performed in view of the simulation of a realistic deployment process of an AAL system. This process includes a setting phase in which the system is trained under factory conditions on environmental configurations that are representative of the possible application conditions, using a minimal set of two room couples which are different between each other in many possible ways by construction. This process led to the collection of Dataset 1 (see Fig. 2.a) and Dataset 2 (see Fig. 2.b). Such a step is then followed by an operational phase in which the already trained system is used in an unseen ambient configuration (though sampled from an environmental framework similar to that one of the first two room pairs) that was unavailable for system training and setting. This process led to the collection of Dataset 3 (see Fig. 2.c).

Figure 2.a to 2.c show that rooms contain typical office furniture, including desks, chairs, metal cabinets, monitors, that are asymmetrically arranged. From the point of view of wireless communications, this is a harsh environment due to the multi-path reflections caused by walls and metal cabinets, and the interference produced by the presence of a large number of electronic devices and wireless networks.

The experimental campaign exploits a WSN comprising 5 IRIS nodes ${ }^{3}$ embedding a Chipcon AT86RF230 radio subsystem that implements the IEEE 802.15.4 standard. Four sensors act as anchors and are deployed in fixed places near the room corners, while one sensor acts as the mobile and is carried by an actor impersonating the user. Each experiment consists in measuring the

3 Crossbow Technology Inc., http://www.xbow.com 


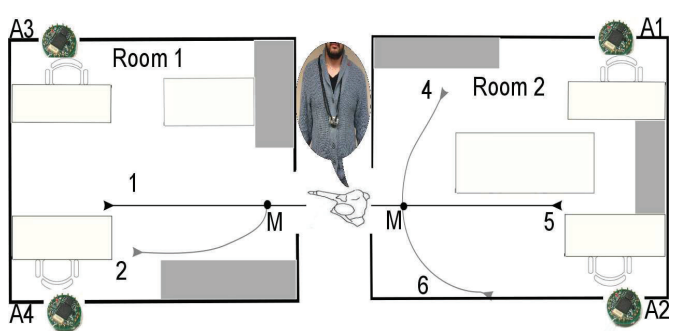

(a) Dataset 1: : Each room is characterized by 2 working positions.

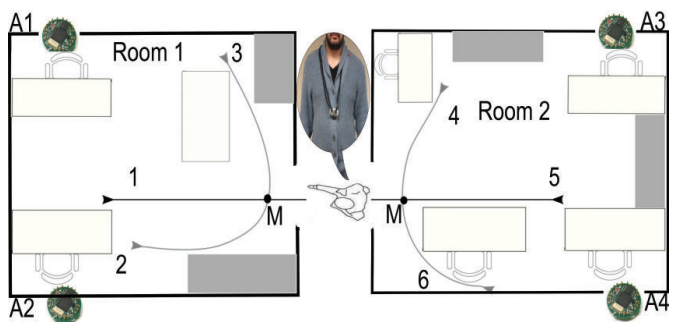

(b) Dataset 2 : Room 1 and room 2 are characterized by 2 and 4 working positions, respectively.

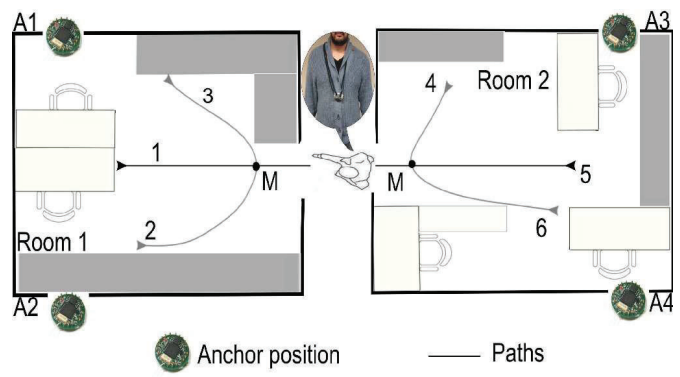

(c) Dataset 3 : Room 1 and room 2 are characterized by 2 and 3 working positions, respectively.

Fig. 2 Room layout and test-bed setup for the movement prediction scenario: (a)-(c) show three different room couples, each corresponding to a separate dataset in the benchmark. The figures highlight the position of the anchors in the rooms (note that in Dataset 1 the achors are positioned differently), as well as the location of the wearable mobile (as highlighted on the bubble, the mobile sensor is worn on the chest of the user). The plot identifies 6 patterns of user movements, some corresponding to a change in the spatial context (i.e. paths 1 and 5), some which conclude in the same room (i.e. paths 2, 3, 4 and 6.).

RSS between the anchors and the mobile for a set of different patterns of user trajectories. The location of the anchors and of the mobile is detailed in Figure 2. The anchors are placed at an height of $1.5 \mathrm{~m}$ from the ground and the mobile is worn on the chest of the actor. The speed of the actor is almost constant and around $1 \mathrm{~m} / \mathrm{s}$. The collected measurements denote RSS samples (integer 
values ranging from 0 to 100) gathered by sending a beacon packet from the anchors to the mobile at regular intervals, 8 times per second, using the full transmission power of the IRIS mote.

The patterns of the user trajectories are also shown in Fig. 2 with arrows numbered from 1 to 6 . Two patterns ( 1 and 5 ) run from Room 1 to Room 2, and viceversa, corresponding to a change in the spatial context of the user, while curved movements (paths 2, 3, 4 and 6) preserve the spatial context. The ground truth data for each user trajectory was obtained by hand-labeling each sequence of RSS measurements according to the possible path types illustrated in Fig.2. Table 2 summarizes the statistics of the patterns for each dataset: due to physical constraints, Dataset 1 does not have a curved movement in Room 1 (path 3). The number of trajectories leading to a room change, with respect to those that preserve the spatial context, is indicated in Table 2 as "Total Change" and "Total Unchanged", respectively. Each path produces a trace of RSS measurements that begins from the corresponding arrow and that is marked when the user reaches a point (denoted with M in Fig. 2) located at $0.6 \mathrm{~m}$ from the door. Overall, the experiment produced about 5000 RSS samples from each of the 4 anchors and for each dataset. The marker $M$ is the same for all the movements, therefore different paths cannot be distinguished based only on the RSS values collected at M.

The experimental scenario and the gathered RSS measures can directly be exploited to formalize a binary classification task on time series for movements forecasting. The RSS values from the four anchors are organized into sequences of varying length (see Table 2) corresponding to trajectory measurements from the starting point until marker M. A target classification label is associated to each input sequence to indicate whether the user is about to change its location (room) or not ${ }^{4}$. In particular, target class +1 is associated to location changing movements (i.e. paths 1 and 5 in Fig. 2), while label -1 is used to denote location preserving trajectories (i.e. paths 2,3,4 and 6 in Fig. 2). The resulting dataset is freely available for download ${ }^{5}$.

In the experiments presented in Section 4.2, the RSS signals were re-scaled to the interval $[-1,1]$. Such re-scaling was performed singularly on the set of traces collected from each anchor in each dataset. Examples of normalized traces, showing the significant noisy characterization of RSS signals, are illustrated in Figure 3.

\subsection{Experimental Results}

Coherently with the realistic deployment of an AAL system discussed in Section 4.1, and with the twofold objective of systematically assessing the pre-

4 Note that for this task, learning has the general aim of distinguishing among the different types of user trajectories (straight or curved in our scenario), based only on the history of noisy RSS signals. On the other hand, the specific application presented in this paper, consisting in the prediction of the user localization (room change or not), represents a concrete example of real-life RC application in the field of AAL.

5 http://wnlab.isti.cnr.it/paolo/index.php/dataset/6rooms 
Table 2 Statistics of the collected user movements. For each dataset it is shown the number of sequences per path type, the number of sequences with positive and negative target classification (Total Changed and Total Unchanged, respectively), and the minimum and maximum length of a sequence in the dataset.

\begin{tabular}{r|c|c|c}
\hline Path Type & Dataset 1 & Dataset 2 & Dataset 3 \\
\hline 1 & \multicolumn{3}{|c}{ Number of Sequences } \\
2 & 26 & 26 & 27 \\
3 & 26 & 13 & 12 \\
4 & - & 13 & 12 \\
5 & 26 & 14 & 13 \\
6 & 13 & 26 & 27 \\
Total Unchanged & 52 & 14 & 13 \\
\hline & \multicolumn{3}{|c}{ Number of Time Steps } \\
Total Changed & 52 & 52 & 54 \\
Lengths min-max & $19-32$ & $34-119$ & $29-129$ \\
\hline
\end{tabular}

dictive performance and appropriately evaluating the generalization ability of the proposed RC system, we consider two binary classification tasks based on the WSN scenario described in Section 4.1. The first task has been designed to exercise the predictive capability of the RC system under a splitting of training/test data that is homogeneous with respect to the given ambient configuration. Indeed, both training and test sequences, although non overlapping, have been sampled from the same group of rooms. Such homogeneous task, comprises all sample trajectories from Datasets 1 and 2, for a total number of 210 RSS sequences. Network performance has been evaluated on this task by holdout validation, whose details are given in the following. The second task has been designed with the objective of assessing the generalization ability of the proposed RC approach in non-homogeneous ambient configurations. Such an heterogeneous task comprises all available data (314 sequences in total), where the union of Datasets 1 and 2 (210 sequences) is used as training set, while Dataset 3 (104 sequences) has the role of an external test set characterized by paths in a different room layout that is completely unseen at the training phase.

In our experiments we used LI-ESN with reservoir dimension $N_{R}$ varying in $\{10,20,50,100,300,500\}, 10 \%$ of connectivity, leaking rate $a=0.1$ and spectral radius $\rho=0.99^{6}$. For reservoir initialization, each non-zero weight value in matrices $\mathbf{W}_{\text {in }}$ and $\hat{\mathbf{W}}$ has been randomly chosen from a uniform distribution over a finite weight alphabet with 8 possible values, uniformly sampled in the range $[-0.4,0.4]$. Note that this weight encoding scheme leads to a memory requirement of just 3 bits for weight memorization. For each reservoir

6 Note that the choice of the connectivity, leaking rate and spectral radius values is not critical for the task. The experiments in this section use the same values identified in previous preliminary works, e.g. Gallicchio et al (2012). 

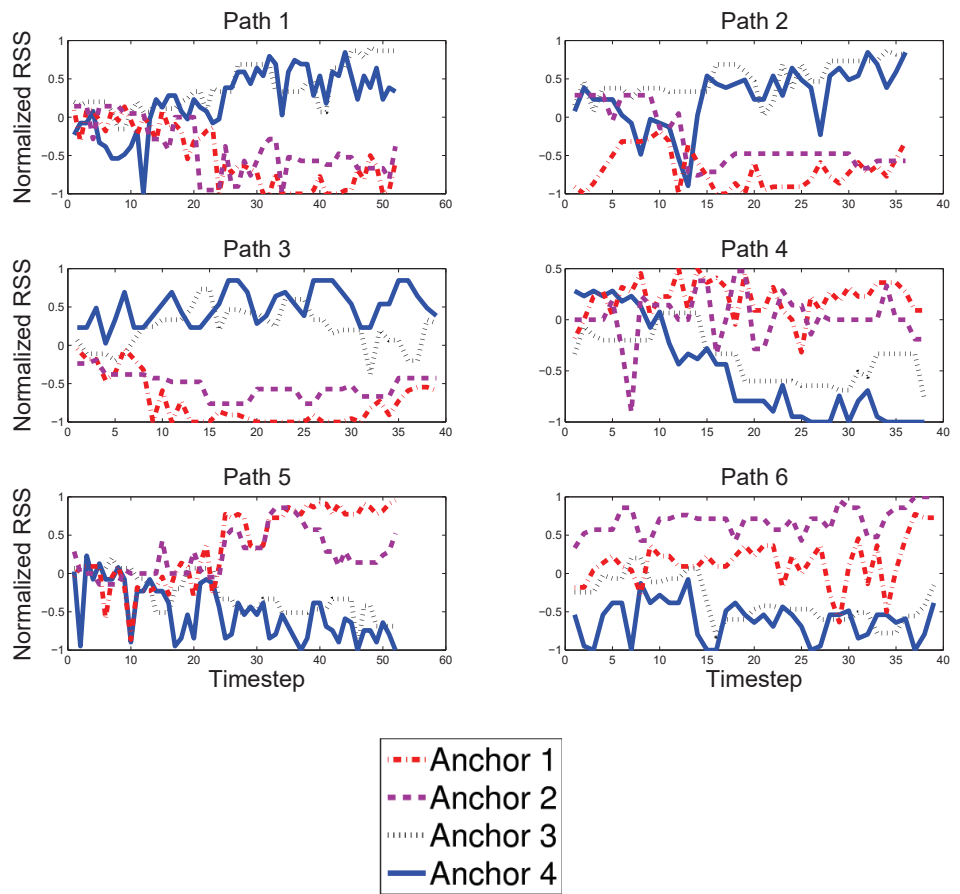

Fig. 3 Examples of normalized RSS traces from the 6 possible movement paths from Dataset 2. Each plot shows the RSS measured for all the 4 anchors in the WSN (see the legend).

hyper-parametrization used, we have independently generated a number of 10 reservoir guesses (and results were averaged over the guesses). The readout of LI-ESNs has been trained by pseudo-inversion and ridge regression, with regularization parameter $\lambda_{r} \in\left\{10^{-1}, 10^{-3}, 10^{-5}\right\}$, which has been selected on a validation set according to the model selection procedure described in the following.

We have considered different modular settings corresponding to the use of 1,2, 3 and 4 anchors, according to the WSN experimental setup scenario described in Section 4.1. Different modular settings correspond to different possible configurations of an RC-based system embedded on the WSN motes. In particular, we have explored a local setting, in which each single LI-ESN receives the RSS input from a single anchor. This configuration corresponds to the case where each LI-ESN module is deployed on-board of a mote of the WSN and receives in input only the RSS signal computed locally to the mote, i.e. there is no connectivity (at the learning-level) among the modules on the different motes. Moreover, we have considered three non-local modular settings in which each LI-ESN receives in input the RSS signal from 2, 3 
and 4 anchors, i.e. each RC module is fed by non-local input data (using all the available anchors in the environment). These latter modular settings are referred to, in the following, as non-local 2, non-local 3 and non-local 4, where the figures refer to the number of anchors made available to the LIESN, i.e. $2,3^{7}$ and 4 , respectively. In this respect, a particularly interesting case is the non-local 4 setting, which corresponds to the case of a complete connectivity among the RC modules available in the WSN deployed for the proposed experimental scenario. Finally, we have taken into consideration a distributed network setting corresponding to an ensemble of local LI-ESN modules. In this case each LI-ESN is fed with the RSS signal from one anchor and the output of the system is obtained by a committee of the local readouts, analogously to a weighted voting scheme among the RC modules on-board the 4 motes in the sensor network. In particular, for each input sequence the output of the ensemble network is the sign of the sum of the outputs of the 4 local readouts.

We have first considered the homogeneous setup, on which, for each reservoir dimension and modularity type (discussed above), we have performed a model selection procedure on the readout regularization. The data splitting for model selection and testing has been designed according to a stratified holdout scheme, with training and test sets containing respectively the $80 \%$ and the $20 \%$ of the available data, and such that $\approx 30 \%$ of the training set has been used as validation set (for model selection). On the heterogeneous task, for each reservoir dimension and network modularity, we have considered the same readout regularization selected on the homogeneous setup for the corresponding configuration. In this case, the RC networks were re-trained on the whole training set of the heterogeneous task (i.e. the union of Datasets 1 and 2) and tested on the external test set (i.e. Dataset 3).

First, we study the results on the homogeneous setup by analyzing the trade-off between predictive accuracy (effectiveness), computed as the average proportion of correctly classified trajectories, and the computational/memory cost (efficiency), as determined by the reservoir size. Figure 4 summarizes the behavior of predictive test accuracy as a function of reservoir size for the three most-interesting modular settings, i.e local, ensemble and non-local 4. Note that Figure 4, also reports the standard deviation corresponding to the local modular setting, computed over the possible different WSN deployments with one anchor. Overall, the proposed approach seems notably effective with predictive accuracy on the test set reaching $97.6 \%$, for the best result, and, globally, over $85 \%$ on all modular settings, including small, non-trivial, reservoir dimensions, i.e. $\geq 20$.

Accuracy scales well with the reservoir size, reaching a saturation point around 100 reservoir units, after which performance stabilizes and no dramatic accuracy increase is observed for a further addition of reservoir units. Such an indication is particularly interesting in view of the embedding of the

\footnotetext{
7 Note that the results reported for the non-local 2 and non-local 3 settings are averaged over all the possible configurations of the available anchors (i.e. 6 configurations for non-local 2 and 4 configurations for non-local 3 ).
} 


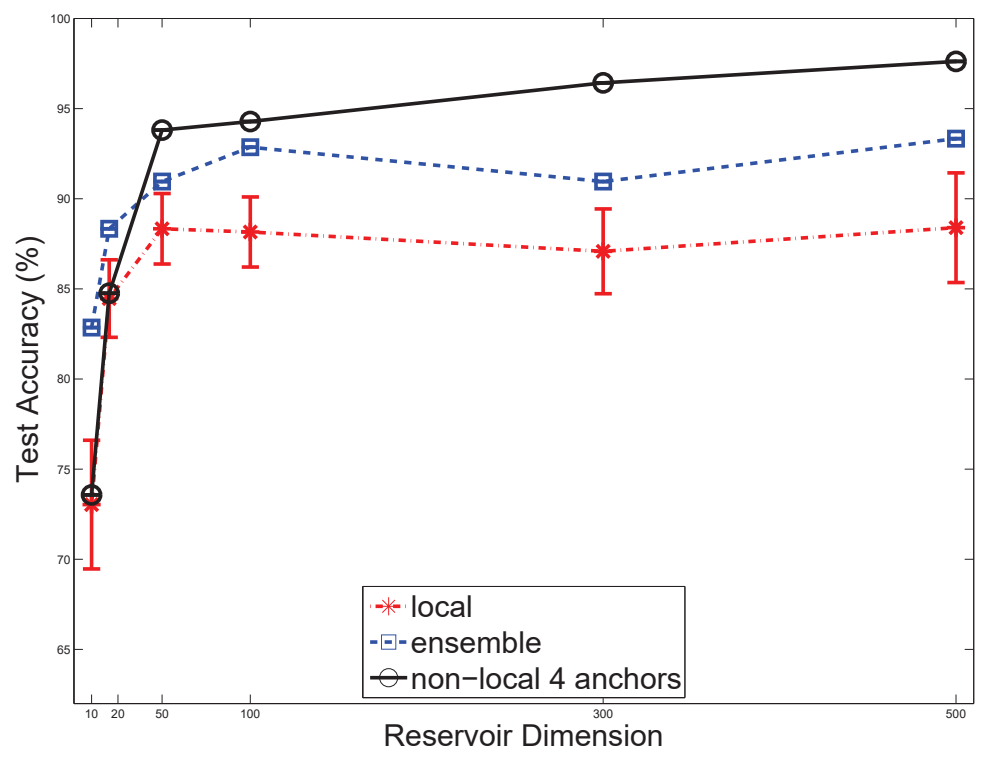

Fig. 4 Averaged test accuracy achieved by LI-ESN on the homogeneous task, in correspondence of the following network settings: local, ensemble and non-local with 4 anchors. Standard deviation, depicted as vertical lines, is computed for the local setting case over the different possibilities of WSN deployments.

RC modules on the WSN nodes, as it suggests that an excellent predictive accuracy can be achieved already with small-to-medium sized reservoirs (i.e. with dimension in the $[50,100]$ range). With respect to the network settings, it is clear that the most informed architecture, i.e. the non-local 4, is performing best since it can exploit and fuse the full information from all the 4 RSSI streams. On the other hand, also the ensemble configuration shows a notable test accuracy, that is comparable to that of the non-local 4 case for mediumsized reservoirs (i.e. for $N_{R}=100$ ensemble achieves $92.9 \%$ accuracy compared to $94.3 \%$ in non-local 4), while showing a neat increase in performance with respect to the purely local configuration that reaches $88.2 \%$ for $N_{R}=100$. Again, this is an interesting indication in the context of WSN embedding: the ensemble configuration has, in fact, the same computational/memory cost of its local counterpart, which is reduced with respect to a non-local 4 model of the same size. Nonetheless, we recall that the ensemble prediction can be straightforwardly obtained by leveraging the underlying wireless communication infrastructure to circulate the predictions of the single local models.

Table 3 shows the detailed results on the homogeneous task, including training and test accuracy for all the modular configurations (and standard deviations over the correspondent different WSN deployments). These results 
Table 3 Training and test average accuracy (and standard deviation) achieved by LI-ESN on the homogeneous task, in correspondence of the following network settings: local, ensemble and non-local with 2, 3 and 4 anchors. Standard deviation is computed over the possible configurations of the corresponding WSN.

\begin{tabular}{|c|c|c|c|}
\hline Modularity Type & $N_{R}$ & $\mathrm{TR}$ & $\mathrm{TS}$ \\
\hline local & $\begin{array}{r}10 \\
20 \\
50 \\
100 \\
300 \\
500\end{array}$ & $\begin{array}{l}70.7( \pm 2.7) \\
84.8( \pm 2.6) \\
89.8( \pm 3.0) \\
92.2( \pm 3.4) \\
91.3( \pm 6.3) \\
93.1( \pm 3.3)\end{array}$ & $\begin{array}{l}73.0( \pm 3.6) \\
84.5( \pm 2.2) \\
88.3( \pm 2.0) \\
88.2( \pm 1.9) \\
87.1( \pm 2.4) \\
88.4( \pm 3.0)\end{array}$ \\
\hline ensemble & $\begin{array}{r}10 \\
20 \\
50 \\
100 \\
300 \\
500\end{array}$ & $\begin{array}{l}82.0( \pm 0.0) \\
90.4( \pm 0.0) \\
93.8( \pm 0.0) \\
96.7( \pm 0.0) \\
97.9( \pm 0.0) \\
96.5( \pm 0.0)\end{array}$ & $\begin{array}{l}82.9( \pm 0.0) \\
88.3( \pm 0.0) \\
91.0( \pm 0.0) \\
92.9( \pm 0.0) \\
91.0( \pm 0.0) \\
93.3( \pm 0.0)\end{array}$ \\
\hline non-local 2 & $\begin{array}{r}10 \\
20 \\
50 \\
100 \\
300 \\
500\end{array}$ & $\begin{array}{l}70.0( \pm 4.2) \\
82.4( \pm 3.3) \\
93.8( \pm 2.7) \\
95.0( \pm 4.2) \\
94.9( \pm 4.0) \\
96.6( \pm 2.3)\end{array}$ & $\begin{array}{l}66.2( \pm 7.1) \\
82.1( \pm 3.8) \\
89.2( \pm 3.2) \\
91.2( \pm 3.8) \\
93.6( \pm 2.2) \\
93.8( \pm 5.0)\end{array}$ \\
\hline non-local 3 & $\begin{array}{r}10 \\
20 \\
50 \\
100 \\
300 \\
500\end{array}$ & $\begin{array}{l}74.4( \pm 2.4) \\
84.4( \pm 1.8) \\
95.6( \pm 1.6) \\
99.2( \pm 0.8) \\
98.3( \pm 2.7) \\
98.8( \pm 0.8)\end{array}$ & $\begin{array}{l}73.0( \pm 4.6) \\
83.3( \pm 2.4) \\
92.4( \pm 2.3) \\
93.8( \pm 0.9) \\
94.1( \pm 1.2) \\
95.6( \pm 1.5)\end{array}$ \\
\hline non-local 4 & $\begin{array}{r}10 \\
20 \\
50 \\
100 \\
300 \\
500\end{array}$ & $\begin{array}{r}76.8( \pm 0.0) \\
85.5( \pm 0.0) \\
96.6( \pm 0.0) \\
100.0( \pm 0.0) \\
100.0( \pm 0.0) \\
100.0( \pm 0.0)\end{array}$ & $\begin{array}{l}73.6( \pm 0.0) \\
84.8( \pm 0.0) \\
93.8( \pm 0.0) \\
94.3( \pm 0.0) \\
96.4( \pm 0.0) \\
97.6( \pm 0.0)\end{array}$ \\
\hline
\end{tabular}

show a graceful degradation of the test accuracy as the number of anchors that provide input to the LI-ESN reduces, with a rough $1 \%$ difference between adjacent number of anchors. The difference between the average accuracy on the training set and on the test data is quite limited, especially if we consider that the training and test trajectories are collected in 4 different rooms, indicating that the proposed approach robustly generalizes its predictions to unseen trajectories from different rooms (corresponding to the 6 prototype paths in Fig. 2).

By taking a step further, we are interested in assessing whether such stability and generalization capabilities still hold when the approach is tested on a completely unknown environment. This can be evaluated through the heterogeneous task, where the ESN models are trained on trajectories from 4 rooms 


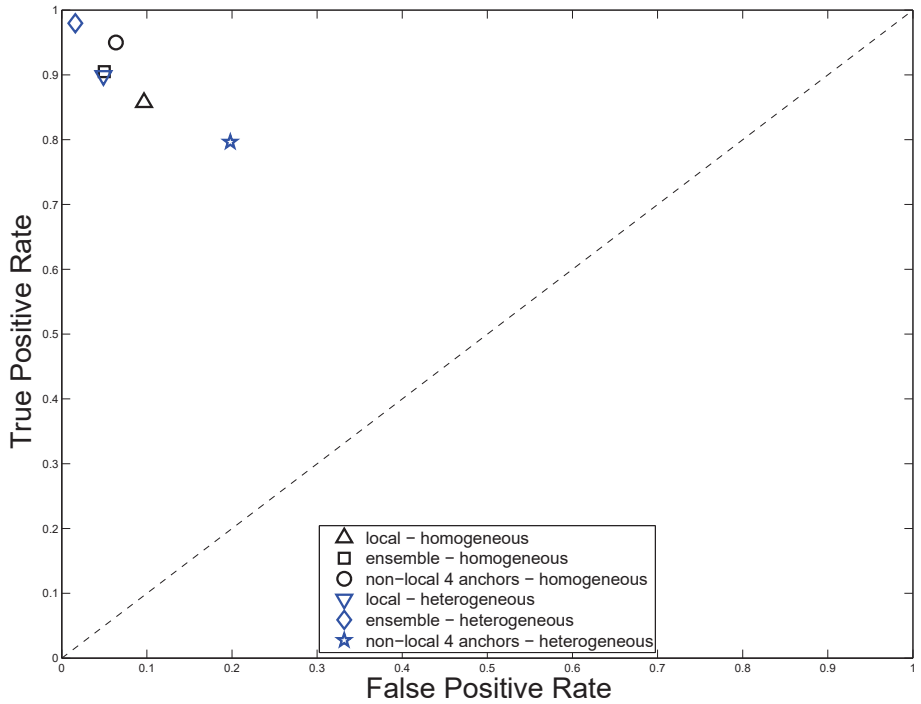

Fig. 5 ROC plot on the test set for LI-ESN with $N_{R}=100$ reservoir units on the homogeneous and heterogeneous tasks, in correspondence of the following network settings: local, ensemble and non-local with 4 anchors.

and tested on samples gathered in 2 new, previously unseen rooms. Figures 5 and 6 show the Receiver Operating Characteristic (ROC) plots comparing the performance of the local, ensemble and non-local 4 configurations on the homogeneous and heterogeneous tasks, using reservoir dimensions of 100 (see Fig. 5) and 500 (see Fig. 6), respectively. These representative reservoir sizes have been chosen given that they are associated with the best overall accuracy (for $N_{R}=500$ ) and with the best accuracy-efficiency tradeoff (for $N_{R}=100$ ). The ROC plots suggest that the results on the heterogeneous task, as a whole, fall roughly within the same ROC area of high-sensitivity/high-specificity as the homogeneous results. A larger variability with respect to the modular configuration can be observed on the heterogeneous task, especially when using less reservoir units (see Fig. 5). Besides this, the results confirm that, overall, the proposed movement forecasting approach can generalize well to unseen environments, which is a key property for ensuring success in real-life deployments.

Full accuracy results (and standard deviations over the possible WSN deployments) on the heterogeneous task, including all modular configurations, are reported in Table 4 . As expected, training accuracy is fully coherent with the homogeneous results in Table 3. On the other hand, the testing accuracy shows a peculiar behavior, where the LI-ESN models using inputs from a single anchor are more accurate than those using 2, 3 and 4 anchors. The ensemble 


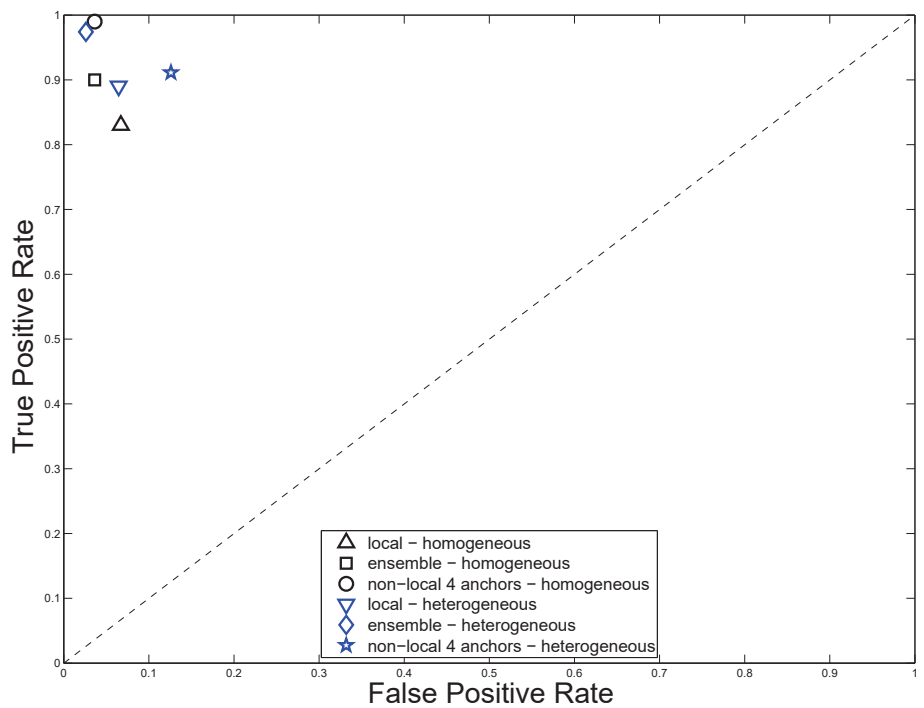

Fig. 6 ROC plot on the test set for LI-ESN with $N_{R}=500$ reservoir units on the homogeneous and heterogeneous tasks, in correspondence of the following network settings: local, ensemble and non-local with 4 anchors.

configuration has the best performance as it builds on the single-anchor models and, coherently with the results in Table 3, it increases the accuracy with respect to the corresponding local solution. We point out that such particular behavior is simply the result of a peculiarity in the testing rooms which, by pure chance, lead to trajectories that are best suited to the single-anchor models. In fact, the robust validation process that we use for model selection and training of the LI-ESN model is totally oblivious on the characteristics of the testing trajectories which, in the heterogeneous case, come from a totally unknown environment. As a result of this, it can happen that certain unknown testing rooms favor one model over the other. Nevertheless, this does not change the overall effectiveness of the approach, that, even in the worst configuration case, reaches predictive accuracies close to the $90 \%$ also in the challenging heterogeneous task.

Overall, the empirical analysis confirms the effectiveness of the proposed RC-based movement forecasting approach, whose generalization capabilities scale well with respect to both unseen trajectories and unknown environments. On the side of computational efficiency, the LI-ESN model is capable of achieving high accuracies already with a small-to-medium number of reservoir units, while using a constrained sparse encoding for the recurrent reservoir and input-to-reservoir weights. We recall that the constrained LI-ESN model used in the experiments is characterized by a finite 3-bits weight alphabet. 
Table 4 Training and test average accuracy (and standard deviation) achieved by LIESN on the heterogeneous task, in correspondence of the following network settings: local, ensemble and non-local with 2, 3 and 4 anchors. Standard deviation is computed over the possible configurations of the corresponding WSN.

\begin{tabular}{|c|c|c|c|}
\hline Modularity Type & $N_{R}$ & $\mathrm{TR}$ & $\mathrm{TS}$ \\
\hline local & $\begin{array}{r}10 \\
20 \\
50 \\
100 \\
300 \\
500\end{array}$ & $\begin{array}{l}71.7( \pm 2.2) \\
85.4( \pm 2.0) \\
89.8( \pm 2.7) \\
91.5( \pm 3.2) \\
90.5( \pm 5.8) \\
92.7( \pm 3.3)\end{array}$ & $\begin{array}{l}82.3( \pm 2.9) \\
95.4( \pm 3.2) \\
95.0( \pm 4.7) \\
92.4( \pm 9.1) \\
90.0( \pm 8.7) \\
91.2( \pm 5.2)\end{array}$ \\
\hline ensemble & $\begin{array}{r}10 \\
20 \\
50 \\
100 \\
300 \\
500\end{array}$ & $\begin{array}{l}82.2( \pm 0.0) \\
90.0( \pm 0.0) \\
93.3( \pm 0.0) \\
96.2( \pm 0.0) \\
97.8( \pm 0.0) \\
97.5( \pm 0.0)\end{array}$ & $\begin{array}{l}96.7( \pm 0.0) \\
99.1( \pm 0.0) \\
98.9( \pm 0.0) \\
98.2( \pm 0.0) \\
96.8( \pm 0.0) \\
97.4( \pm 0.0)\end{array}$ \\
\hline non-local 2 & $\begin{array}{r}10 \\
20 \\
50 \\
100 \\
300 \\
500\end{array}$ & $\begin{array}{l}69.2( \pm 4.5) \\
83.2( \pm 2.8) \\
94.0( \pm 2.4) \\
95.4( \pm 3.6) \\
95.4( \pm 3.6) \\
97.0( \pm 1.5)\end{array}$ & $\begin{array}{l}61.5( \pm 4.3) \\
74.1( \pm 4.3) \\
84.9( \pm 3.8) \\
89.3( \pm 3.9) \\
88.5( \pm 5.9) \\
88.3( \pm 7.8)\end{array}$ \\
\hline non-local 3 & $\begin{array}{r}10 \\
20 \\
50 \\
100 \\
300 \\
500\end{array}$ & $\begin{array}{l}74.5( \pm 2.6) \\
84.9( \pm 1.5) \\
95.6( \pm 1.5) \\
99.0( \pm 1.2) \\
98.6( \pm 2.3) \\
98.7( \pm 1.0)\end{array}$ & $\begin{array}{l}61.9( \pm 3.2) \\
66.7( \pm 3.8) \\
78.2( \pm 1.6) \\
83.9( \pm 1.1) \\
87.4( \pm 3.2) \\
89.7( \pm 3.6)\end{array}$ \\
\hline non-local 4 & $\begin{array}{r}10 \\
20 \\
50 \\
100 \\
300 \\
500\end{array}$ & $\begin{array}{r}76.3( \pm 0.0) \\
86.2( \pm 0.0) \\
96.8( \pm 0.0) \\
99.9( \pm 0.0) \\
100.0( \pm 0.0) \\
100.0( \pm 0.0)\end{array}$ & $\begin{array}{l}63.7( \pm 0.0) \\
70.0( \pm 0.0) \\
76.4( \pm 0.0) \\
79.9( \pm 0.0) \\
86.9( \pm 0.0) \\
89.3( \pm 0.0)\end{array}$ \\
\hline
\end{tabular}

More concretely, if we consider a LI-ESN model with a reservoir dimension $N_{R}=100$, an arithmetic limited to 16 bits float representation, we have that the total memory occupation, on each mote, of the recurrent reservoir plus the input-to-reservoir weight matrices is 1452 bytes, for the local/ensemble configuration, and 1565 bytes for the non-local 4 configuration. In a standard LI-ESN model, without finite-alphabet encoding, we would have a memory occupation per mote of 3402 bytes for local/ensemble and about 4002 bytes for the non-local 4 configuration, which is almost doubled with respect to the proposed constrained model. More importantly, given the numbers above, the constrained ensemble LI-ESN with 100 reservoir units would realistically fit into the memory of a standard wireless mote, that is typically limited to $8-10$ Kbytes, paving the way for an effective embedded implementation of the RSS- 
based movement forecasting solution described in this Section. We also observe that for larger reservoirs, e.g. for $N_{R}=500$, in correspondence of a marginal improvement of the predictive performance, the memory occupation costs of our lightweight scheme reaches approximatively 38 Kbytes for both the $l o$ $\mathrm{cal} /$ ensemble and non-local 4 configurations. The memory requirement would be even greater for the standard LI-ESN memorization techniques, in the order of 80 Kbytes, overall resulting in unsuitable configurations for practical embedding on typical WSN motes. The latter observations, and the predictive performance results discussed in this Section, suggest that a reservoir dimension of $N_{R}=100$ can be sufficient to address real-world AAL tasks of the type of the one presented in this paper. In particular, for the task on user movement forecasting at hand, in view of the embedding the proposed solution on a real WSN, such choice of the reservoir dimensionality shows that it is possible to find a particularly suitable and practical solution from the point of view of the tradeoff between predictive performance and computational feasibility (i.e. memory and computational costs).

\section{Conclusions}

We have discussed the potential of the efficient RC approach in building effective embedded solutions for AAL applications using WSN technologies. We have identified a real-world predictive challenge related to user movement forecasting using noisy RSS information gathered through a small but realistic WSN.

The proposed RC approach has been empirically assessed based on realworld data gathered in such experimental scenario. A complete analysis has been presented, taking into consideration the efficacy of the model in terms of predictive accuracy, as well as the tradeoff with computational efficiency, measured through memory occupation resulting from varying reservoir dimensions. The experimental results point out that the proposed LI-ESN approach achieves very accurate predictions of the user spatial context without resorting to a large (over 100 units) reservoir, while showing robustness to variations to the monitored indoor ambient. In particular, the approach is capable of generalizing its predictions both to unseen user trajectories and to novel testing environments, i.e. unknown at training time. Such capability is of paramount importance for the development of practical AAL applications, as it allows to consistently reduce the installation and setup costs of the solution. For instance, we envisage a scenario in which an ESN-based movement forecasting system is trained, e.g. in laboratory/factory, on RSS measurements captured on sample rooms and, then, deployed and put into operation into its target environment, reducing the need for an expensive configuration phase.

Further, we have proposed and empirically assessed several modular architectures comprising single or multiple RC modules with various degrees of mutual interaction, from total independence to full RSS information sharing, passing across intermediate ensemble-based architectures. While full informa- 
tion sharing yields to the best overall accuracy, the experimental results also point out the effectiveness of an ensemble-based solution, that comprises RC modules performing independent predictions on separate input information and weakly collaborating to achieve a consensus prediction. Both modular configurations are well suited to be deployed on a WSN, since the single LIESN can be embedded into the motes and can interact through the WSN communication service, either to share RSS information or to achieve the consensus. In this respect, the computational efficiency of the LI-ESN solution gains fundamental importance, given the resource-constrained nature of WSN nodes and the reactivity required to deliver timely predictions. To this end, particular care has been taken to define a parsimonious LI-ESN model which builds on a sparse reservoir with a limited number of units and a constrained weight alphabet, strongly reducing the memory occupation due to the reservoir, without significantly negatively impacting the predictive accuracy.

Overall, the results of the empirical analysis suggest that LI-ESN has an excellent trade-off between accuracy, generalization and efficiency, when dealing with noisy time series data. As such, it can be considered a good candidate for the development of a distributed learning system for AAL applications that embeds the LI-ESN modules directly on the wireless sensor nodes. As part of the research effort of the EU FP7 RUBICON project, we plan to develop such distributed learning infrastructure for WSNs (Bacciu et al, 2012), founding on the LI-ESN approach discussed in this paper. This learning system is intended to provide adaptive movement forecasting and localization service in WSNs, as well as more general event-recognition and prediction services for AAL applications. Finally, as a side contribution of this work, we have defined an effective movement prediction benchmark, providing a freely-available dataset ${ }^{8}$ comprising extensive real-world RSS measurements.

Acknowledgements This work is partially supported by the EU FP7 RUBICON project (http://www.fp7rubicon.eu/), contract n. 269914.

\section{References}

AAL (2009) Ambient assisted living roadmap. URL http://www .aaliance. eu/public/documents/aaliance-roadmap/

Antonelo EA, Schrauwen B, Campenhout JMV (2007) Generative modeling of autonomous robots and their environments using reservoir computing. Neural Proc Lett 26(3):233-249

Antonelo EA, Schrauwen B, Stroobandt D (2008) Event detection and localization for small mobile robots using reservoir computing. Neural Networks 21(6):862-871

Bacciu D, Gallicchio C, Micheli A, Chessa S, Barsocchi P (2011) Predicting user movements in heterogeneous indoor environments by reservoir com-

8 http://wnlab.isti.cnr.it/paolo/index.php/dataset/6rooms 
puting. In: Bhatt M, Guesgen HW, Augusto JC (eds) Proc. of the IJCAI Workshop on Space, Time and Ambient Intelligence (STAMI) 2011, pp 1-6

Bacciu D, Chessa S, Gallicchio C, Lenzi A, Micheli A, Pelagatti S (2012) A general purpose distributed learning model for robotic ecologies. In: To appear in the Proceedings of the 10th International IFAC Symposium on Robot Control (SYROCO'12)

Bahl P, Padmanabhan V (2000) RADAR: an in-building Rf-based user location and tracking system. In: Proc. of INFOCOM 2000. 19th Annual Joint Conf. of the IEEE Comp. and Comm. Soc., vol 2, pp 775-784 vol.2, DOI 10.1109/ INFOCOM.2000.832252

Barsocchi P, Lenzi S, Chessa S, Giunta G (2009a) A novel approach to indoor rssi localization by automatic calibration of the wireless propagation model. In: IEEE 69th Vehicular Tech. Conf., pp 1-5, DOI 10.1109/VETECS.2009. 5073315

Barsocchi P, Lenzi S, Chessa S, Giunta G (2009b) Virtual calibration for rssibased indoor localization with ieee 802.15.4. In: 2009 IEEE Int. Conf. on Comm. ICC '09., pp 1-5, DOI 10.1109/ICC.2009.5199566

Battiti R, Villani A, Le Nhat T (2002) Neural network models for intelligent networks: deriving the location from signal patterns. In: Proceedings of AINS2002, Citeseer

Boedecker J, Obst O, Mayer N, Asada M (2009) Initialization and selforganized optimization of recurrent neural network connectivity. HFSP Journal 3(5):340-349

Brunato M, Battiti R (2005) Statistical learning theory for location fingerprinting in wireless lans. Comput Netw ISDN Syst 47(6):825-845, URL http://dx.doi.org/10.1016/j.comnet.2004.09.004

Büsing L, Schrauwen B, Legenstein R (2009) Connectivity, dynamics, and memory in reservoir computing with binary and analog neurons. Neural Computation 22(5):1272-1311

Chang M, Terzis A, Bonnet P (2009) Mote-based online anomaly detection using echo state networks. In: Krishnamachari B, Suri S, Heinzelman W, Mitra U (eds) Distributed Computing in Sensor Systems, Lecture Notes in Computer Science, vol 5516, Springer Berlin/Heidelberg, pp 72-86

EvAAL (2011) The 1st evaal competition, evaluating aal systems through competitive benchmarking. Special theme on Indoor Localization and Tracking, AAL Forum proceedings, valencia, ES

Fette G, Eggert J (2005) Short term memory and pattern matching with simple echo state networks. In: Proceedings of the International Conference on Artificial Neural Networks (ICANN), Springer, Lecture Notes in Computer Science, vol 3696, pp 13-18

Gallicchio C, Micheli A (2010) A markovian characterization of redundancy in echo state networks by PCA. In: Proceedings of the European Symposium on Artificial Neural Networks (ESANN) 2010, d-side, pp 321-326

Gallicchio C, Micheli A (2011) Architectural and markovian factors of echo state networks. Neural Networks 24(5):440 - 456, DOI DOI:10.1016/j. neunet.2011.02.002 
Gallicchio C, Micheli A, Barsocchi P, Chessa S (2012) User movements forecasting by reservoir computing using signal streams produced by mote-class sensors. In: Mobile Lightweight Wireless Systems (Mobilight 2011), Lecture Notes of the Institute for Computer Sciences, Social Informatics and Telecommunications Engineering, vol 81, Springer Berlin Heidelberg, pp $151-168$

Gärtner T (2003) A survey of kernels for structured data. SIGKDD Explorations Newsletter 5:49-58

Hajnal M, Lorincz A (2006) Critical echo state networks. In: Proceedings of the International Conference on Artificial Neural Networks (ICANN) 2006, pp 658-667

Hartland C, Bredeche N (2007) Using echo state networks for robot navigation behavior acquisition. In: IEEE Int. Conf. on Robotics and Biomimetics, IEEE, pp 201-206

Jaeger H (2001) The "echo state" approach to analysing and training recurrent neural networks. Tech. rep., GMD - German National Research Institute for Computer Science

Jaeger H, Haas H (2004) Harnessing nonlinearity: Predicting chaotic systems and saving energy in wireless communication. Science 304(5667):78-80

Jaeger H, Lukosevicius M, Popovici D, Siewert U (2007) Optimization and applications of echo state networks with leaky- integrator neurons. Neural Networks 20(3):335-352

Kolen J, Kremer S (eds) (2001) A Field Guide to Dynamical Recurrent Networks. IEEE Press

Kontkanen P, Myllymaki P, Roos T, Tirri H, Valtonen K, Wettig H (2004) Topics in probabilistic location estimation in wireless networks. In: Personal, Indoor and Mobile Radio Communications, 2004. PIMRC 2004. 15th IEEE International Symposium on, vol 2, pp 1052 - 1056 Vol.2, DOI 10.1109/ PIMRC.2004.1373859

Kushki A, Plataniotis K, AN Venetsanopoulos AN (2007) Kernel-based positioning in wireless local area networks. IEEE Trans Mobile Comp 6(6):689 -705, DOI 10.1109/TMC.2007.1017

Legenstein RA, Maass W (2007) Edge of chaos and prediction of computational performance for neural circuit models. Neural Networks 20(3):323-334

Liu H, Darabi H, Banerjee P, Liu J (2007) Survey of wireless indoor positioning techniques and systems. Systems, Man, and Cybernetics, Part C: Applications and Reviews, IEEE Transactions on 37(6):1067 -1080, DOI 10.1109/TSMCC.2007.905750

Lukosevicius M, Jaeger H (2009) Reservoir computing approaches to recurrent neural network training. Computer Science Review 3(3):127 - 149

Luo X, OBrien WJ, Julien CL (2011) Comparative evaluation of received signal-strength index (rssi) based indoor localization techniques for construction jobsites. Advanced Engineering Informatics 25(2):355 - 363

Maass W, Natschlager T, Markram H (2002) Real-time computing without stable states: a new framework for neural computation based on perturbations. Neural Computation 14(11):2531-60, DOI 10.1162/089976602760407955 
Oubbati M, Kord B, Palm G (2010) Learning robot-environment interaction using echo state networks. In: Proc. of the 11th int. conf. on Simul. of adapt. behavior, Springer-Verlag, SAB'10, pp 501-510

Pan JJ, Kwok J, Yang Q, Chen Y (2006) Multidimensional vector regression for accurate and low-cost location estimation in pervasive computing. IEEE Tran Knowl and Data Eng 18(9):1181 -1193, DOI 10.1109/TKDE.2006.145

Rodan A, Tiňo P (2011) Minimum complexity echo state network. IEEE Transactions on Neural Networks 22(1):131 -144

Steil JJ (2004) Backpropagation-decorrelation: online recurrent learning with $\mathrm{O}(\mathrm{N})$ complexity. In: Proceedings of the IEEE International Joint Conference on Neural Networks (IJCNN) 2004, vol 2, pp 843-848

Steil JJ (2006) Online stability of backpropagation-decorrelation recurrent learning. Neurocomputing 69(7-9):642-650

Tiňo P, Dorffner G (2001) Predicting the future of discrete sequences from fractal representations of the past. Machine Learning 45(2):187-217

Tiňo P, Cernanský M, Benusková L (2004) Markovian architectural bias of recurrent neural networks. IEEE Transactions on Neural Networks 15(1):615

Verstraeten D, Schrauwen B, D'Haene M, Stroobandt D (2007) An experimental unification of reservoir computing methods. Neural Networks 20(3):391403

Waegeman T, Antonelo E, Wyffels F, Schrauwen B (2009) Modular reservoir computing networks for imitation learning of multiple robot behaviors. In: 8th IEEE Int. Symp. on Comput. Intell. in Robotics and Autom., IEEE, pp $27-32$

Wu CL, Fu LC, Lian FL (2004) Wlan location determination in e-home via support vector classification. In: Networking, Sensing and Control, 2004 IEEE International Conference on, vol 2, pp 1026 - 1031 Vol.2

Youssef M, Agrawala A (2005) The horus wlan location determination system. In: MobiSys'05: Proc. of the 3rd Int. Conf. on Mobile Sys. appl. and services, ACM, New York, NY, USA, pp 205-218, DOI http://doi.acm.org/10.1145/ 1067170.1067193

Zàruba GV, Huber M, Kamangar FA, Chlamtac I (2007) Indoor location tracking using RSSI readings from a single wi-fi access point. Wireless Netw (13):221,235 